\title{
Cracks in the curriculum: an appreciation
}

There is a crack, a crack in everything. That's how the light gets in. "Anthem" - Leonard Cohen

$\mathrm{M}$ any academics and clinicians like us, who teach in medical schools do not have formal training in education, yet that does not mean that we are not effective teachers, or reflective about how we teach.

The perspective of one of the coauthors [GF] is that teaching is storytelling: I tell a story about a topic and what the end result is depends on how well I tell the story and how receptive the audience is to the telling. What results is unpredictable. Some audiences are engaged by the subject matter, get involved in the storytelling and the result is something wonderful. Others don't engage, wait for the whole sorry mess to end and thank God when it does. Even within these constraints, the teaching product varies widely because it isn't just a piece of knowledge we are handing out, it is a perspective on their career that should have a lasting impact that, with each student, will be different.

The perspective of the other coauthor [JC] is somewhat analogous: in my teaching I try to inculcate that there is not always a right answer or a straight trajectory that can be followed - a result is that my classes are open-ended and learning outcomes unpredictable. Sometimes this frustrates students (my favourite criticism of my teaching was from a student who wrote on a course evaluation that I set a goal of getting students to think about being a doctor and about the profession of medicine, but this student wanted "something more concrete" as an objective).

Our point is that we need to retain a bit of space for lateral thinking in the curriculum so that students may engage in their own reflective moments about the frequent "uncertainty" of medicine. But we are afraid that we might "learning outcomes/objectives" it out of existence to the detriment of our students' education. If we wish to be serious about medical

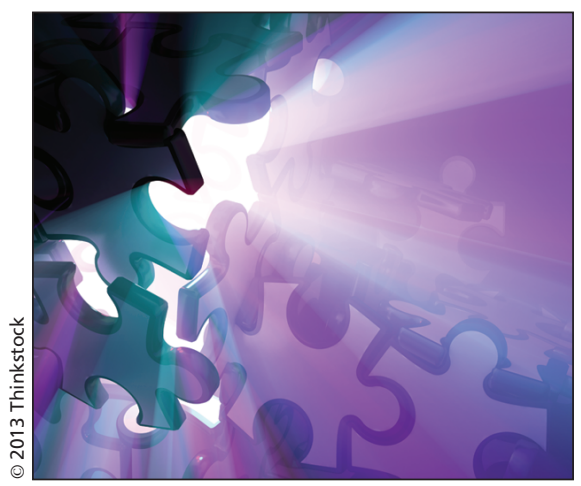

education, and if we aspire to promote scholarship in our future physicians, then we must not centre our curriculum exclusively on the linear thinking of outcomes and objectives. Some teaching modalities may not be measurable in the short-term, but an apparent absence of effectiveness metrics is not sufficient reason to eschew or devalue these methods.

We recognize that undergraduate medical training (as distinct from education) is very much driven by learning objectives and outcomes (e.g., CanMEDS, LCME, CACMS, MCC). There is a requirement to insert such measures in a professional curriculum to ensure that teaching is in alignment with the dictates of accrediting and licensing bodies. Yet, there may be unintended consequences if objectives and outcomes are followed unthinkingly or too slavishly in the classroom. A recent British critique that has been widely circulated in Canadian academic circles entitled Learning Outcomes are Corrosive ${ }^{1}$ declaims the demerits of learning outcomes as they "threaten to disrupt the conduct of the academic relationship between teacher and student," "foster a climate that inhibits the capacity of students and teachers to deal with uncertainty," "devalue the art of teaching" and finally that the "regime of learning outcomes ... breeds a culture of cynicism and irresponsibility." Underpinning these criticisms is the notion that such "utilitarian education" reduces the intrinsic meaning of learning at the university level and tends to oversimplify material, while rewarding "those who have internalized template-speak." Worse yet, it may promote among academics a "calculating and instrumental attitude where responsibility becomes equated with box-ticking" because the emphasis switches more to achieving outcomes without actually gauging what students have learned.

There is, we feel, a movement within society (and maybe the profession) to regard physicians (and physicians-to-be) as the result of a cookie cutter; they should all think, look and behave in a specified and predictable way. Learning objectives and outcomes, to our collective mind, are a derivative of that; in effect, a type of spoon-feeding that privileges unreflective behaviour. We believe that many medical students (but admittedly not all) are hungry for a more nutritious intellectual diet that encourages an attentive attitude that may translate to better bedside manner and professionalism. A routine faculty teaching evaluation of one of us $[\mathrm{JC}]$ recently revealed first-year students' responses to nonspoon-feeding, humanities-based pedagogy: "Made me think of what being a doctor means from my perspective and from society's perspective," "Classes were refreshingly relaxed" and "I was engaged ... and found myself enjoying lectures on things that I previously thought I would not particularly enjoy." Let us be clear that we subscribe to a curriculum that is imbued with technical knowledge, rigour and clinical skills, but perhaps the odd curricular crack occasioned by dint of circumstance, or by design, is to be appreciated. Often, cracks are how the light gets in.

\section{J.T.H. Connor PhD}

Gerard Farrell MD

Faculty of Medicine

Memorial University

St John's, NL

\section{Reference}

1. Furedi F. Learning outcomes are corrosive. CAUT Bulletin 2013;60. Available: www.cautbulletin.ca/en _article.asp?articleid=3575 (accessed 2013 May 31).

CMAJ 2013. DOI:10.1503/cmaj.130373

All editorial matter in CMAJ represents the opinions of the authors and not necessarily those of the Canadian Medical Association. 